\title{
Heavy-light and light-light weak matrix elements on the lattice *
}

\author{
Claude W. Bernard \\ Department of Physics, CB1005, \\ Washington University, \\ St. Louis, MO 63130, USA
}

I review recent developments in lattice weak matrix element calculations. I focus on on $f_{B}$ (both with propagating quarks and in the static limit for the $b$ quark), semi-leptonic form factors for $D$ meson decay, form factors for $B \rightarrow K^{*} \gamma$, and $B_{K}$.

\section{INTRODUCTION}

The main obstacle to precise determinations of the parameters of the Standard Model is the difficulty in calculating hadronic matrix elements of weak operators. The wide range in allowed values for hadronic quantities such as $f_{B}$ (the pseudoscalar decay constant of the $B$ meson), the form-factors for semi-leptonic $B$ decay, and $B_{K}$ (describing mixing in the $K-\bar{K}$ systems) results in large uncertainties in our determinations of the Cabibbo-Kobayashi-Maskawa angles and $m_{t}$ [1]. Lattice gauge theory, at least in principle, should allow us to compute such matrix elements from first principles, i.e., with control on all sources of systematic error. Here, I review the current status of this effort.

The talk is organized as follows. I first discuss $f_{B}$, both in the static approximation [2] and with propagating quarks. In the case of $f_{B}^{\text {stat }}$, the results show a wide spread, both among various groups working on the problem, and as a function of the lattice spacing $a$. I explain the reasons for the spread and show that the $a$-dependence seems to be understandable in perturbation theory. The variation among groups is likely due to the poor signal-to-noise properties of staticlight propagators. This can introduce spurious dependences on the details of how the signal is extracted. I discuss in particular the quality of the data obtained with different types of "smearing." In the case of $f_{B}$ with propagating quarks, the results (at least the raw data) from different groups seem rather better in agreement. The main issues which I consider are the proper normalization of quarks with $m a \sim 1$, the consistency between Wilson quarks and Sheikholeslami-Wohlert (SW) improved quarks, and the consistency between the propagating and static decay constants.

Recent results for the form factors for semileptonic decays of $D$ mesons are then discussed [3]. I focus on the normalization factors for the local and point-split currents and describe how their mass dependence may be understood, at least qualitatively. This provides a way to reconcile (or at least reduce the discrepancies between) results from different currents with different heavy quark masses.

I then describe results presented at this conference for the form factors of the rare decay $B \rightarrow K^{*} \gamma$. This is a promising new way for the lattice to provide a handle on the Standard Model. The results suggest that rather stringent constraints on the Standard Model could be obtained with modest improvements in the lattice calculations and/or the experiment.

Finally I review some exciting new developments in the computation of $B_{K}$ on the lattice. All systematic errors but one appear to be under control, and that one is a chiral loop effect which should be no larger than a few percent. The result, $B_{K}(\mu=2 \mathrm{GeV})=0.616 \pm 0.020 \pm 0.017$ or

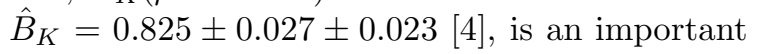
contribution of lattice QCD to Standard Model phenomenology. 
Table 1

Results for $f_{B}^{\text {stat }}$ from various groups

\begin{tabular}{|c|c|c|}
\hline group & $\beta$ & $f_{B}^{\text {stat }}(\mathrm{MeV})$ \\
\hline FNAL[5] & 5.7 & $305(15)$ \\
& 5.9 & $269(20)$ \\
& 6.1 & $228(21)$ \\
& 6.3 & $237(22)$ \\
\hline BLS[6] & 6.3 & $235(20) \pm 21$ \\
\hline APE[0] 8$]$ & 6.0 & $350(40) \pm 30$ \\
& $6.0[$ clover] & $370(40)$ \\
\hline UKQCD[9] & $6.0[$ clover] & $286_{-10-42}^{+8}+67$ \\
& $6.2[$ clover] & $253_{-15-14}^{+16+105}$ \\
\hline P-W-C[10] & $5.74,6.0,6.26$ & $230(22) \pm 26$ \\
& (extrap.) & \\
\hline Hashimoto[11] & $6.0[$ nrqcd] & $320(30) \pm 60$ \\
\hline
\end{tabular}

\section{2. $f_{B}^{\text {stat }}$}

Table 1 gives some results for $f_{B}^{\text {stat }}$ from various groups. Note that the values range from a high of $370 \pm 40 \mathrm{MeV}$ to a low of $228 \pm 21 \mathrm{MeV}$. However, it is misleading to compare these final answers, because different groups use 1) different values for $Z_{A}^{\text {stat }}$, the lattice renormalization constant for the static-light axial vector current, and 2) different methods to set the lattice scale, $a$. Since what is really being calculated is $(f \sqrt{m})^{\text {stat }} a^{\frac{3}{2}}$, the result for $f_{B}^{\text {stat }}$ is particularly sensitive to the value used for $a$.

In order to make a more realistic comparison, I therefore fix the scale uniformly by using computations [12] of the string tension, $\sigma$, and plot in Fig. 11 the "raw" value of $(f \sqrt{m})^{\text {stat }} \sigma^{-\frac{3}{4}} v s$. $a \sqrt{\sigma}$. Here, "raw" means before multiplying by $Z_{A}^{\text {stat }}$, except that in the case of the SW action, I do correct for the difference between $Z_{A}^{\text {stat }}$ with the Wilson and SW actions by using perturbation theory [13, 14] with a boosted coupling [15] $g^{2}=1.77$ at $\beta=6.0$ and $g^{2}=1.65$ at $\beta=6.2$.

Two features stand out in Fig. 1. First, there are real disagreements between the groups. For the moment, I will focus on the lower tier of results (including the FNAL and the BLS points). I do this not only because it includes my own work [6], but also because the FNAL group uses the best sources [16,17]. (The UKQCD results are somewhat higher, but only because of the rela-

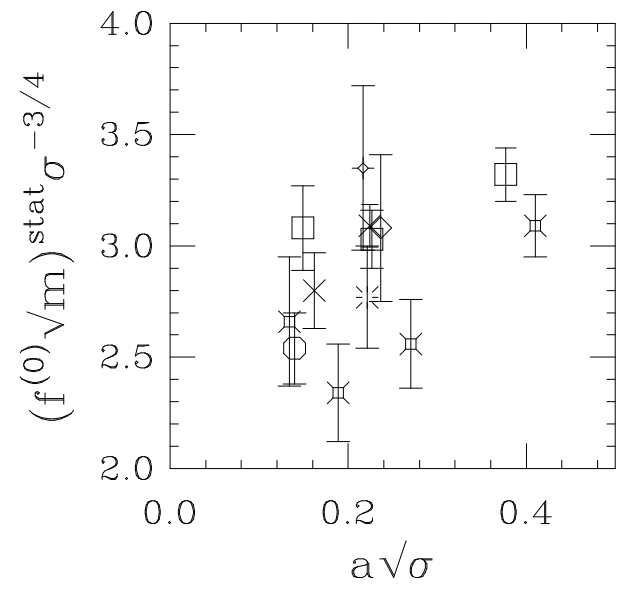

Figure 1. Raw value of $\left(f m^{\frac{1}{2}}\right)^{\text {stat }} \sigma^{-\frac{3}{4}}$ vs. $a \sigma^{\frac{1}{2}}$ from various groups; only the relative perturbative correction between SW and Wilson quarks is included. Statistical errors only. Some points have been moved slightly horizontally for clarity. Key: FNAL fancy square; BLS octagon; UKQCD (SW action) cross; P-W-C square; APE diamond; APE (SW action) fancy diamond; Hashimoto burst. For references see Table 1.

tive perturbative correction for the SW action.) The second feature is the strong $a$ dependence of the lower results. This is puzzling at first glance. If the cause were the presence of higher dimension operators $(\mathrm{O}(a)$ effects), one would expect that that the SW $(\mathcal{O}(a)$ improved) points would show significantly less scale dependence, which does not appear to be the case. Furthermore, the results for propagating quarks seem to show considerably smaller scaling violations. (Of course, the size of statistical errors makes definitive statements difficult.) I believe, however, that most, if not all, of the $a$ dependence can actually be explained by the "improved perturbation theory" ideas of Lepage and Mackenzie.

\subsection{Tadpole improved perturbation the- ory and the static limit}

Lepage and Mackenzie 15 argue that bare lattice perturbation theory is ill-behaved because 1) the bare lattice coupling is a poor choice of an expansion parameter, and 2) lattice tadpoles are large. They cure the first disease by using a more 
physical coupling constant such as $g_{V}$, which is defined in terms of the heavy-quark potential. The second disease is cured by summing up the tadpoles with a mean field theory approach: they show that the simple replacement of the link $U$ by its mean value $u_{0}$ accounts for the bulk of the perturbative corrections to many quantities. For example, for Wilson fermions, the replacement $U \rightarrow u_{0}$ just changes the hopping parameter $\kappa$ to $\tilde{\kappa} \equiv \kappa u_{0}$. The resulting free field theory has a critical hopping parameter $\tilde{\kappa}_{c}=\frac{1}{8}$ which would imply $\kappa_{c}=\frac{1}{8 u_{0}}$, a relation which agrees reasonably well with simulations when one defines $u_{0} \equiv\left\langle\frac{1}{3} \operatorname{tr} U_{\text {plaq }}\right\rangle^{\frac{1}{4}}$. In the following, I will define $u_{0}$ by $\kappa_{c}$ for Wilson quarks; that is, I take

$u_{0} \equiv \frac{1}{8 \kappa_{c}}=1-0.109 g^{2}+\ldots$.

In computing some perturbative quantity, the prescription for "mean-field improvement" (or "tadpole improvement") is then to take out explicit powers of $u_{0}$ (depending on the number of links which enter the definition of the object), replace them by the value of $u_{0}$ in the simulation $\left(1 /\left(8 \kappa_{c}\right)\right)$, and remove the appropriate power of $1-0.109 g^{2}+\ldots$ from the remaining perturbative expansion. For example, the 1-loop renormalization factor for the local axial current with Wilson fermions in the chiral limit is [18] $\tilde{Z}_{A}=2 \kappa_{c}\left(1-0.133 g^{2}\right)$, where the ${ }^{\sim}$ on $\tilde{Z}_{A}$ implies that I include the standard factor $2 \kappa_{c}$ relating Wilson to continuum fermions. The tadpoleimproved prescription would then be

$\tilde{Z}_{A} \rightarrow 2 u_{0} \kappa_{c} \frac{1-.133 g^{2}}{1-.109 g^{2}}=\frac{1}{4}\left(1-.024 g_{V}^{2}\left(\frac{1}{a}\right)\right)$

The factor of $u_{0}$ which is removed is really two factors of $\sqrt{u_{0}}$ coming from wave function renormalization on the external lines. The small coefficient in front of $g_{V}^{2}$ indicates that improved perturbation theory is working well [19]. The scale $(1 / a)$ at which $g_{V}^{2}$ is evaluated is a typical one for non-tadpole diagrams; when the quadratically divergent tadpoles are left in, the scale is typically closer to $\pi / a$.

An interesting feature of perturbative quantities in the static approximation, however, is that they are much less tadpole-dominated than the corresponding quantities with Wilson quarks. This can easily be understood by examining the static action:

$$
\begin{array}{r}
S_{s}=\sum_{x} \bar{h}_{x}\left(\frac{1+\gamma_{0}}{2}\right)\left[h_{x}-U_{0}^{\dagger}(x-\hat{0}) h_{x-\hat{0}}\right] \\
\rightarrow \sum_{x} \bar{h}_{x}\left(\frac{1+\gamma_{0}}{2}\right)\left[h_{x}-u_{0} h_{x-\hat{0}}\right]
\end{array}
$$

From eq. (4) one can easily calculate, using a hopping expansion, the mean field theory static propagator. We get

$G_{s}(x, y)=\delta_{\vec{x}, \vec{y}}^{(3)} e^{\left.-\left(\ln u_{0}^{-1}\right)\left(t_{x}-t_{y}\right)\right)}$

This shows that $u_{0}$ contributes to mass renormalization $\left(\ln u_{0}^{-1}\right)$, but not wave function renormalization, since there is no overall factor in $G_{s}$.

Equivalently, in momentum space one has $G_{s}(p)=\left(1-u_{0} e^{i p_{0}}\right)^{-1}$. The $u_{0}$ factor looks like wave function renormalization at first glance. However, expanding about the pole, we let $p_{0}=$ $i \ln u_{0}+p_{0}^{\prime}$ and find $G_{s}\left(p_{0}^{\prime}\right)=\left(1-e^{i p_{0}^{\prime}}\right)^{-1}$, which still has residue 1 . Thus, unlike the Wilson case, there is no tadpole contribution to the wave function renormalization of heavy quarks; one can also see this explicitly in the perturbative calculations 13.

In the case of the static-light axial current renormalization constant, one then takes out only a factor of $\sqrt{u_{0}}$ for the light quark, and divides by $1-\frac{.109}{2} g^{2}$. Using the known perturbative results 13.14, I then get, in the chiral limit for the light quark,

$\tilde{Z}_{A, \text { stat }}^{W} \rightarrow\left(\frac{1}{2}\right)\left[1-g_{V}^{2}\left(q^{*}\right)(.135-\delta)\right]$

$\tilde{Z}_{A, \text { stat }}^{S W} \rightarrow \sqrt{\frac{\kappa_{c}^{S W}}{4 \kappa_{c}^{W}}}\left[1-g_{V}^{2}\left(q^{*}\right)(.090-\delta)\right]$

where the superscripts " $W$ " and " $S W$ " on $\tilde{Z}_{A, \text { stat }}$ indicate Wilson or SW light quarks, respectively, $\delta \equiv .025 \ln \left(a m_{B}\right)$, and $q^{*}$ is the scale at which $g_{V}^{2}$ should be evaluated. The static renormalization constants are clearly not tadpole dominated, since the coefficients of $g_{V}^{2}$ in the above equations are relatively large (compare eq. (2)).

It is not completely clear what scale $q^{*}$ to put into eqs. (6),(7). In cases where the scale after removing tadpoles has been estimated using 
the method of ref. [15], it is $\lesssim 1 / a$ : for $\kappa_{c}$, one has $q^{*}=1.03 / a$ [15]; for two non-relativistic QCD (NRQCD) masses, one has $q^{*}=0.81 / a$ and $0.67 / a$ 20]. In the current case, the scale is estimated to be $2.36 / a$ before tadpole removal [21], but it may not be reduced as much as in the other cases by removing the tadpoles, since the tadpoles play a less important role. Indeed, since the time when this talk was given, the non-tadpole scale has been estimated by Hill and Hernandez [22]; their preliminary result is $q^{*}=2.18 / a$. Note that $q_{V}^{2}(2.18 / a)$ is quite large, ranging from 2.8 at $\beta=5.7$ to 1.9 at $\beta=6.3$. This makes the term $g_{V}^{2}\left(q^{*}\right)(.135-\delta)$ range from .26 to .23 ; with $q^{*}=1 / a$, the corresponding numbers are .42 to .31. The higher order perturbative effects could therefore be quite large, perhaps $10 \%$ or even more, which implies considerable uncertainty in $f_{B}^{\text {stat }}$.

In Figs. 2 and 3, I put in the tadpole improved corrections to the raw static numbers, using $q^{*}=1 / a$ and $q^{*}=2.18 / a$ respectively. Note that with $q^{*}=1 / a$, the $a$ dependence of the the lower tier of results is small. With $q^{*}=2.18 / a$, there is still may be significant $a$ dependence, but it is reduced from Fig. 1, and the results are consistent with a constant if one drops the point at largest $a(\beta=5.7)$. One is now tempted to extrapolate to $a=0$. With either scale, a linear extrapolation of the FNAL and BLS results gives $(f \sqrt{m})^{\text {stat }} \sigma^{-\frac{3}{4}} \approx 1.6 \pm 0.2$ at $a=0$, which would imply $f_{B}^{\text {stat }} \approx 190 \pm 25 \mathrm{MeV}$. Extrapolation of the UKQCD (SW-action) points would produce almost the same answer. Clearly, however, there are large systematic errors in the result at this stage. I estimate that total systematic error associated with the extrapolation, with the setting of the scale, with the uncertainty in the perturbative corrections, and with the extraction of the raw numbers themselves (see below) is roughly twice the statistical error.

\subsection{Smearing}

I now come to the issue of the large variation in the results from different groups. I believe this stems from the intrinsically poor signal-to-noise ratio for static-light propagators at large times [23,24, 16]. The problem has been dealt with by

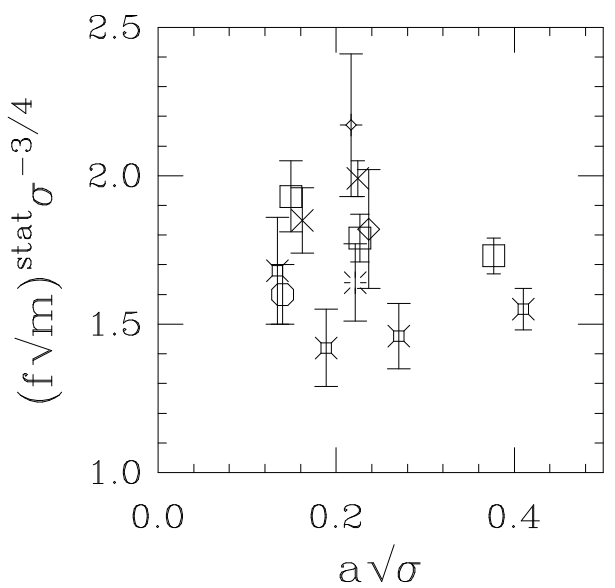

Figure 2. Same as Fig. 1 but including the tadpole improved perturbative corrections, with $g_{V}^{2}$ evaluated at scale $q^{*}=1 / a$.

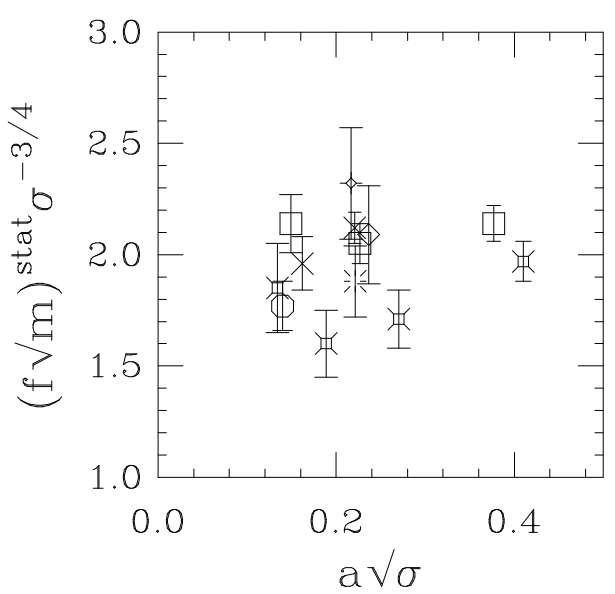

Figure 3. Same as Fig. 2 but at scale $q^{*}=2.18 / a$.

smearing the static quark (or both quarks) in the static-light sources. This can improve the situation in two ways: 1 ) by producing a better overlap with the ground state, thereby moving the plateau in to smaller times where the signal-tonoise is better, and 2) by increasing the statistics. The second point, which has not always been appreciated in the literature, will be discussed below.

$f_{B}^{\text {stat }}$ is computed from the asymptotic behavior 
of the correlation functions

$$
\begin{array}{r}
G_{L S}(t)=\sum_{\vec{x}}\left\langle 0\left|A_{0}(\vec{x}, t) \chi_{S}^{\dagger}(\overrightarrow{0}, 0)\right| 0\right\rangle \\
"=" \sum_{\vec{x}}\left\langle 0\left|\chi_{S}(\vec{x}, t) A_{0}^{\dagger}(\overrightarrow{0}, 0)\right| 0\right\rangle, \\
G_{S S}(t)=\sum_{\vec{x}}\left\langle 0\left|\chi_{S}(\vec{x}, t) \chi_{S}^{\dagger}(\overrightarrow{0}, 0)\right| 0\right\rangle,
\end{array}
$$

where $A_{0}=\bar{h} \gamma_{\mu} \gamma_{5} q$ is the local or almost local (i.e., point split) axial current ( $h$ and $q$ are the static and light quark fields, respectively), and $\chi_{S}$ is the smeared meson interpolating operator:

$\chi_{S}(\vec{x}, t)=\sum_{\vec{y}} f(\vec{y}) \bar{h}(\vec{x}+\vec{y}, t) \gamma_{5} q(\vec{x}, t)$,

with $f(\vec{y})$ some smearing function. (I assume here that only the static quark is smeared, which is the most common practice.)

I write " =" in eq. (9) because the two correlators, while equal when averaged over an infinite number of configurations, have different statistical errors with a finite number of configurations. The smeared-source, local-sink, eq.(8), is considerably less noisy than the local-source, smearedsink, eq.(9). This was noticed with the first use of static-light smearing 25]; a nice comparison of the results from the two appears in the recent UKQCD paper [9].

The reason for the difference in statistics is easy to understand. Since the static quark propagates along a straight line in the time direction, only the static propagator from $(\overrightarrow{0}, 0)$ to $(\overrightarrow{0}, t)$ (coming only from terms with $\vec{x}=-\vec{y}$ in eq. (9)) contributes when the source is local. Independent of what the smearing function is, the result just depends on a single product of links in each configuration, and hence has large fluctuations. When the source is smeared, many products of links enter into the result from each configuration, and the fluctuations are thereby greatly reduced.

Note that the statistics issue is completely independent of the the relative ground-state vs. excited-stated overlap for a given interpolating field. This means there may be a certain amount of tradeoff between good overlap and good statistics. For example, wall sources, in which both the static quark and the light quark are smeared over
Figure 4. APE data for the effective mass from the smeared-smeared correlator for various smearing sizes, $L_{S}$. $\beta=6.0$, SW action, 210 configs.

the entire lattice, clearly have poor overlap, since the mean distance between the static and light quarks is much larger than the size of the ground state. However, they have potentially very good statistics since all time-like link products on the lattice are used.

Figs. 14 and 5 show data for the $G_{S S}$ effective mass and for $G_{L S} / G_{S S}$ from APE [7] with the SW action at $\beta=6.0$. The heavy quark is smeared over a cube of side $L_{S}$; various values of $L_{S}$ are shown. $G_{L S}$ is calculated with localsource, smeared-sink (eq. (9)), which accounts the comparatively large errors in Fig. 5 (compared with, say, the UKQCD data [9]), despite the large number of configurations (210). I find it difficult to see consistent, convincing plateaus in both $G_{S S}$ and $G_{L S} / G_{S S}: L_{S}=7$ and $L_{S}=9$ are reasonable but $L_{S}=5$ is somewhat doubtful in the former; while only $L_{S}=5$ is convincing in the latter. One really needs plateaus for the same value of $L_{S}$ in both correlators to be able to extract an answer. The APE group takes the variance between the $L_{S}=5$ and the $L_{S}=7$ results as an estimate of the systematic error here; however I am not convinced that this is a good estimator of the error.

Fig. 6 shows the effective mass for various smearings in $G_{S S}$ from the PSI-Wuppertal- 
Figure 5. APE data for the ratio $G_{L S} / G_{S S}$ for various smearing sizes, $L_{S} . \beta=6.0$, SW action, 210 configs.

CERN group (P-W-C) [10]. I do not find the plateau, which they consider to start at $t=3$, very convincing. Since their analysis determines the mass from this correlator and then uses it in determining the location of the plateau in $G_{L S}$, I suspect there may be considerable systematic error involved here. I note, however, that the $\mathrm{P}-\mathrm{W}-\mathrm{C}$ group has varied the parameters of their analysis and does not find large variations in the results. A possible additional source of systematic error arises from the rather small size of the lattice here $\left(18^{3} \times 48\right.$ at $\left.\beta=6.26\right)$.

Some of the difficulty in extracting $f_{B}^{\text {stat }}$ from simulations is illustrated in fig. 7 from our group [6]. The data, at $\beta=6.0$ with cube smearings of side $L_{S}=9$, is fit in two different ways: The dashed lines show simultaneous fits to $G_{S S}$ and $G_{L S}$ in the range $t=(5,10)$; while the solid lines show simultaneous fits to $G_{S S}$ in the range $(3,7)$ and $G_{L S}$ in the range $(9,13)$. While both sets of fits appear, at first glance, reasonably convincing, they give vastly different results. Indeed $f_{B}^{\text {stat }}$ extracted from the first (dashed-line) fits is $\approx 1.5$ times larger than that extracted from the second (solid-line) fits. For this reason we have not felt confident of quoting a result for $f_{B}^{\text {stat }}$ at $\beta=6.0$. Note that, although the number of configurations is small (8), the statistical errors in the data with our sources are at least as small as those in the
Figure 6. P-W-C data for the effective mass from $G_{S S}$, for various smearings. $18^{3} \times 48, \beta=6.26$, $\kappa_{\text {light }}=.1492,43$ configs.

data of the previously discussed groups.

Fig. 8 shows our data [6] with wall sources at $\beta=6.3$. Note that the errors in $G_{L S}$ are very small, but that a two-exponential fit is required because the relative ground-state overlap is poor. For $G_{S S}$, the errors are much larger, because smearing at the sink does not buy anything in terms of statistics, but just adds noise coming from large separations between the quarks. These features are all consistent with the discussion above.

Our data with cube sources at $\beta=6.3$ are shown in Fig. 9. The errors in $G_{L S}$ are larger than with the wall sources, but the ground state overlap is better, and a good plateau is seen. The plateau for $G_{L L}$ is less convincing, but it does have a mass which is consistent with that of the other channel (the the channels are fit simultaneously). We feel safer with the cube sources than with the walls because we can use singleexponential fits, and we extract our final results from cubes of side $L_{S}=15$. Note, however, that the wall sources, as well as a range of different cube sizes, give consistent results. This data set is also stable under change in fitting intervals; unlike the $\beta=6.0$ case, such shifts change the results by only $\approx 3 \%$.

Data from the FNAL group [5] is shown in Fig. 10. The sources used here are close to ideal: 


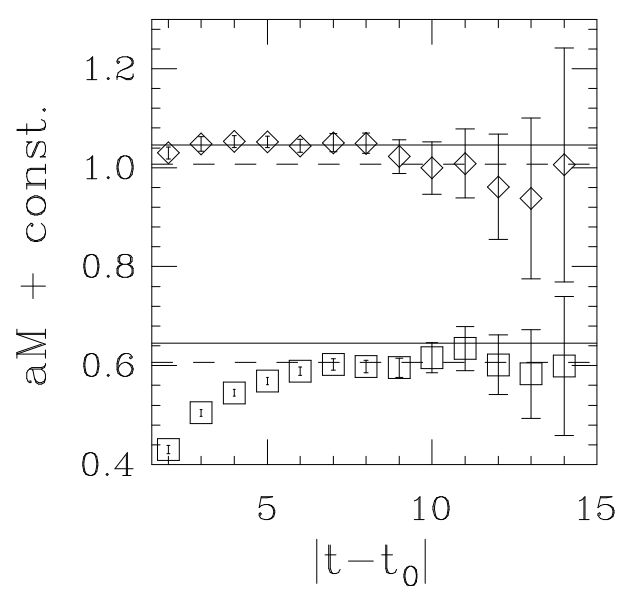

Figure 7. BLS data for the effective mass from $G_{S S}$ (diamonds) and $G_{L S}$ squares. $24^{3} \times 39, \beta=$ $6.0, \kappa_{\text {light }}=.155,8$ configs. The diamonds have been displaced by +0.4 for clarity. The dashed lines and solid lines are different simultaneous fits to the correlators; see text.

their multistate fitting method [16,17] gives a source which is nearly the ground state wavefunction. Despite this, however, it appears that the results could be changed appreciably by moving the fitting intervals. Admittedly, this is not a "typical" but more like a "worst case" example. However, it should serve to emphasize that important systematic errors associated with extracting the ground state exist in the data of all the groups. It will probably take an order of magnitude better statistics (and good sources) to be really confident that this systematic is controlled.

Two new developments discussed at this conference show some promise. First, Draper and McNeile [26] have devised a method of constructing static-light meson sources which is complementary to the FNAL approach but produces similarly near-ideal sources. They show some preliminary data with very nice plateaus and are also able to extract cleanly the wave functions for excited as well as ground states. It will be interesting to see how well they can determine $f_{B}^{\text {stat }}$. Second, two groups 27,11] are using NRQCD for the heavy quark in the heavy-light system. They seek to take advantage of the improved signal-to-noise ratio [23] for NR-light, as opposed to static-light,

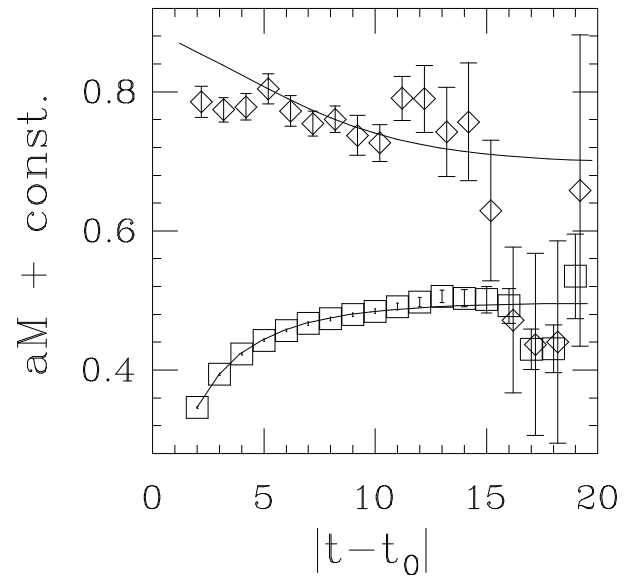

Figure 8. BLS effective masses with wall sources at $\beta=6.3,24^{3} \times 55,20$ configs. (40 sources), $\kappa_{\text {light }}=.150$. Diamonds: $G_{S S}$ (displaced +0.2 for clarity); squares: $G_{L S}$. A simultaneous twoexponential fit to both channels is used. The range is $(5,13)$ for $G_{S S} ;(3,12)$ for $G_{L S}$.

mesons. The results are quite encouraging: their effective mass plots are clearly better than staticlight ones with comparable sources. Hashimoto evaluates of $f_{B}^{\text {stat }}$ (see Table 11); Davies et al. extract $f_{B}$ itself (see below).

The NRQCD group 28] has also carried through a very nice calculation of the $b$-quark mass. They get $m_{b}=4.7 \pm 0.1 \mathrm{GeV}$. This has an important implication for the static theory. It has been suggested [29] that the $1 / m_{Q}(Q$ is a generic heavy quark) corrections to the static limit would not be computable due to the presence of power-law divergences, which could induce large non-perturbative effects. The success of the NRQCD calculation of $m_{b}$, which involves perturbative calculations of such power-law divergences and has several cross-checks, indicates that the concerns expressed in ref. [29] are not likely to be a problem in practice.

I have one final remark on $f_{B}^{\text {stat }}$. Given the issues relating to smearing and $a$ dependence which were discussed above, I was dismayed to find the following comment made in ref.[7]:

"The result of [BLS - ref. [6]] is much lower than all other results obtained at a fixed value of $a$. A possible explanation may be found in the fact that 


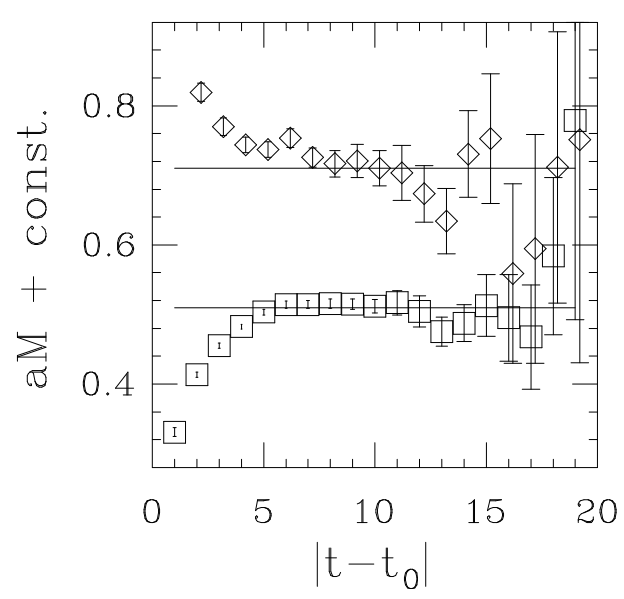

Figure 9. Same as Fig. 8 but the sources are cubes $\left(L_{S}=13\right)$ and a simultaneous single exponential fit in the range $(9,16)$ is used.

in [ref. [6] the results were obtained using very large smearings (or the wall source), which the authors believe to be the most coupled to the lightest state, in contrast with the findings of the present work and of [the FNAL group - ref. [30]]."

We have clearly stated, at least three times [24, 31, 60, the trade-off between ground-state overlap and statistics which motivate our use of wall sources or "very large" smearings (which appear so large only when compared to the small smearings necessitated by the use of eq. (9) in [7]).

\section{3. $f_{B}$ WITH PROPAGATING QUARKS}

Since to approach the $b$ quark on currently available lattices one needs quark masses with $m a \sim 1$, it is necessary to study how lattice quarks behave when the graininess of the lattice is comparable to their Compton wavelength. Kronfeld and Mackenzie 32 33 have initiated such a program. For zero momentum quarks the idea is quite simple. In a free theory the $\vec{p}=0 \mathrm{Wilson}$ propagator is easily shown to be:

$$
\begin{array}{r}
G_{\text {latt }}(t)=\frac{1}{2 \kappa e^{a M_{1}}}\left(\frac{1+\gamma_{0}}{2}\right) e^{-a M_{1} t} \\
=\frac{1}{2 \kappa e^{a M_{1}}} G_{\mathrm{cont}}(t),
\end{array}
$$

where the "pole mass" $M_{1}$ is related to the Lagrangian mass $m_{0}$ by $a M_{1}=\ln \left(1+a m_{0}\right)$, with
Figure 10. Effective masses for $G_{L S}$ (above) and $G_{S S}$ from the FNAL group. $\beta=5.9,16^{3} \times 32$, $\kappa_{\text {light }}=.157,48$ configs.

$a m_{0}=1 /(2 \kappa)-4$ for $r=1$, and $G_{\text {cont }}$ is the continuum $\vec{p}=0$ propagator. The factor $2 \kappa e^{a M_{1}}$ can also be written as $1-6 \kappa$, and it is therefore clear that the correct normalization for a massive free quark field is $\sqrt{1-6 \kappa}$, rather than the $\sqrt{2 \kappa}$, which is only valid near the massless limit.

Interactions can easily be taken into account in the limit of tadpole dominance. In that case, one just replaces $\kappa$ by $\tilde{\kappa} \equiv \kappa u_{0}=\kappa /\left(8 \kappa_{c}\right)$ (see discussion around eq. (11)). In the tadpole approximation, one thus has a normalization factor of $\sqrt{1-6 \tilde{\kappa}}$ for each Wilson quark field in the limit $\kappa \rightarrow 0\left(a M_{1} \rightarrow \infty\right)$.

What are the corrections? First of all there are ones of $\mathcal{O}\left(p^{2}\right)$. These are small if $M_{1} \gg|\vec{p}|$, which should be the case for heavy-light mesons (for which $|\vec{p}| \sim \Lambda_{\mathrm{QCD}}$ ) on current lattices (for which $a M_{1} \sim 1$ implies $M_{1} \gg \Lambda_{\mathrm{QCD}}$ ). A systematic improvement procedure, similar to that of NRQCD, can then take these higher order corrections in to account. Second, there are $\mathcal{O}\left(g^{2}\right)$ corrections. These are small if tadpole dominance is a good approximation. Some early perturbative results seem to bear this out 33. However, recall that tadpole dominance does not work well in the static limit, so one expects some fairly large perturbative corrections ( $\sim 20 \%$ to $30 \%)$ to show up somewhere.

The SW action is a systematic improvement 
on the Wilson action, eliminating terms of $\mathcal{O}(a)$ (but not $\mathcal{O}\left(g^{2} a\right)$ ). One therefore expects it to include the $\mathcal{O}\left(a M_{1}\right)$ difference between the massless normalization $(\sqrt{2 \kappa})$ and the large mass normalization $\left(\sqrt{2 \kappa e^{a M_{1}}}\right)$. This is indeed the case. In the usual SW implementation, one "rotates" the quark field by

$\psi \rightarrow\left(1+\frac{a \not D}{2}\right) \psi=\left(1+\frac{a m_{0}}{2}\right)+\mathcal{O}\left(a^{2}\right)$.

One therefore automatically includes a factor which equals $\sqrt{e^{a M_{1}}}$ up to $\mathcal{O}\left(\left(a m_{0}\right)^{2}\right)$. However, my suggestion to those using the SW action would be to use the exact $(g \rightarrow 0, \kappa \rightarrow 0)$ normalization instead. This is "equivalent" in that its difference with what they are doing already is higher order $\left(\mathcal{O}\left(a^{2}\right)\right)$, but it guarantees that the static limit will be recovered in the $M_{1} \rightarrow \infty$ limit (up to $\left.\mathcal{O}\left(g^{2}\right)\right)$.

Two groups [8.9] have presented evidence that the use of the $\sqrt{1-6 \tilde{\kappa}}$ norm brings the Wilson fermion results into closer agreement with the SW results, as expected. For example, Fig. 11 (9) shows $f_{P} \sqrt{M_{P}}(P$ is a generic heavy-light pseudoscalar) for Wilson quarks with the $\sqrt{1-6 \tilde{\kappa}}$ normalization, and with the ("naive") $\sqrt{2 \kappa}$ normalization, in comparison with the SW results. The naive results vanish exponentially as $M_{P} \rightarrow$ $\infty$, as expected from eq. (13), and therefore cannot be consistent with the static theory. The $\sqrt{1-6 \tilde{\kappa}}$ results are in good agreement with the SW results over the whole range of meson masses examined; this is actually somewhat surprising, since the two are only supposed to agree up to terms of $\mathcal{O}\left(\left(a m_{0}\right)^{2}\right)$. On the other hand, the $\mathrm{P}-\mathrm{W}-\mathrm{C}$ group [34] has presented results showing that $f_{P}$ is less dependent on the lattice spacing if the naive, rather than the $\sqrt{1-6 \tilde{\kappa}}$, normalization is used, although both extrapolate to the same value as $a \rightarrow 0$. I do not understand the reason for these results. It is possible that the naive normalization may accidentally scale better in some limited range of $M_{P}$, but it is clear, as Fig. 11 illustrates, that the naive normalization is completely incorrect as $M_{P} \rightarrow \infty$, and must not scale with $a$ in that limit.

With the $\sqrt{1-6 \tilde{\kappa}}$ normalization, the results with propagating Wilson quarks seem to be in
Figure 11. Comparison, from the UKQCD group, of results for $f_{p} M_{P}^{\frac{1}{2}}$ vs. $1 / M$. Key: SW action (circle); Wilson action with $(2 \kappa)^{\frac{1}{2}}$ normalization (diamond); Wilson action with $(1-6 \tilde{\kappa})^{\frac{1}{2}}$ normalization (square).

good agreement with the static result. Fig. 12 shows the comparison between the two methods from our calculations at $\beta=6.3[6]$. The fit to both results has an excellent $\chi^{2}$. Fig. 13 is a similar comparison from UKQCD [9] at $\beta=6.0$ with SW propagating quarks. Here the solid line fit to the open circles and the static point looks less good, but, because of the correlations between the propagating quark points, it is actually not too bad: $\chi^{2} /$ dof $=1.5$. The fit would improve if tadpole-improved perturbation theory were used for the renormalization factors $Z_{A}$ of both the static and propagating axial currents. For SW propagating quarks, $Z_{A}$ actually is larger after tadpole improvement; while in the static case it is reduced. In Fig. 13, I have attempted to show how the points would move after tadpole improvement. There is some uncertainty here since it is not completely clear how to apply tadpole improvement in the SW case (e.g., whether to use the Wilson or SW $\kappa_{c}$ - there is considerable difference). However it is clear that the agreement between static and propagating will get better. Use of tadpole improvement should also reduce the large uncertainty in scale in the UKQCD results, which is due to a low value of $f_{\pi}$. Tad- 


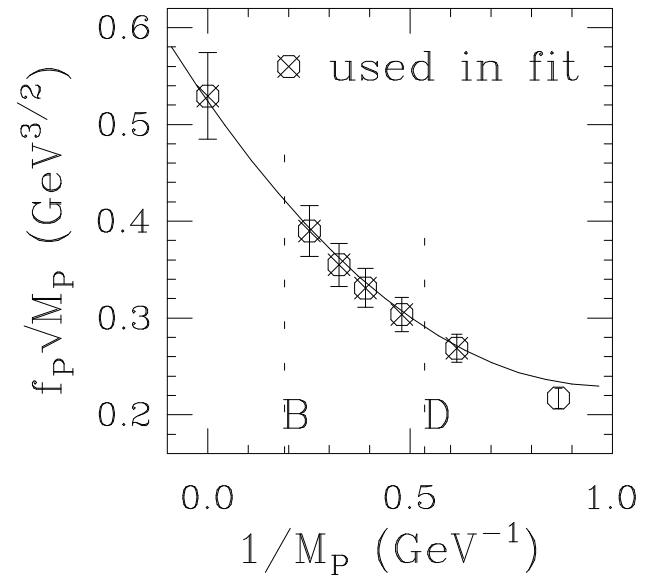

Figure 12. The combined (propagating and static) analysis for $f_{P} M_{P}^{\frac{1}{2}} /\left(1+\frac{\alpha_{s}}{\pi} \ln \left(a M_{P}\right)\right)$ at $\beta=6.3$ from the BLS group. The propagating quarks are normalized with $(1-6 \tilde{\kappa})^{\frac{1}{2}}$. The solid line is a covariant fit, quadratic in $1 / M_{P}$, to the marked points, and has $\chi^{2} /$ dof $=1.9 / 3$. If the renormalization were done exactly as advocated in sec. 2.1, the static point would move down $3 \%$ (using $q^{*}=2.18 / a$ ); the propagating point, $1.5 \%$ (using $q^{*}=1 / a$ ).

pole improvement will bring up the corresponding renormalization constant, making it closer to the non-perturbative value of $\sim 1.09$ [35]. Note also that a determination of $f_{B}$ using non-relativistic heavy quarks (on the same UKQCD configurations) gives a result consistent with the other two methods [27].

In early work [36], fits of static and propagating results seemed to show agreement between the two approaches, even though the naive $\sqrt{2 \kappa}$ normalization was used in the propagating case. However, such fits were non-covariant; they did not take into account the correlations in the propagating-quark data. In ref. [6], we show that a reasonable-looking non-covariant fit can be made between the static point and moderately heavy, naively normalized, a propagating results. However, once correlations are included, the fit looks very bad and has $\chi^{2} /$ dof $=16 / 2$. Clearly: (a) results with naively normalized propagating quarks are inconsistent with the static approach, and (b) covariant fits with meaningful $\chi^{2}$ are cru-
Figure 13. The combined analysis for $f_{P} M_{P}^{\frac{1}{2}} /(1+$ $\left.\frac{\alpha_{s}}{\pi} \ln \left(a M_{P}\right)\right)$ at $\beta=6.0$ from UKQCD. SW propagating quarks are used. The dashed line is a fit to the open circles and the open square is its intercept at $1 / M_{P}=0$. The solid line is the fit with the static point (cross) included. The solid circles show my estimate for the central values if tadpole improvement were used.

cial when testing the consistency of various approaches.

I also call attention to the recent determinations of heavy-light decays constants in full (unquenched) QCD [37]. The results are generally consistent with the quenched results, although at heavy mass (close to the $B$ ) there tends to be some disagreement (the full QCD results are higher). This is not likely to be the effect of quenching, especially since the dynamical quark masses used are not very light. Rather, current computer limitations force the full QCD calculations to be done at rather strong coupling. Near the $B, m a$ can then be quite large, and the $\mathcal{O}\left(p^{2}\right)$ corrections mentioned above can become quite important. In particular, the coefficient of $p^{2}$ in the kinetic energy is no longer $1 /\left(2 M_{1}\right)$, where $M_{1}$ is the "pole mass," but $1 /\left(2 M_{2}\right)$ [32]. Since $M_{2} \gg M_{1}$ for large $M_{1} a$, the full QCD points actually correspond to considerably heavier meson mass. If this effect were put in, the disagreement between quenched and full results would likely go away. Note that the correction $M_{1} \rightarrow M_{2}$ has already been applied to the data in ref. [6]. 
Table 2

Results for $f_{B}$ and $f_{D}$ from various groups

\begin{tabular}{|c|c|c|}
\hline group & $f_{B}(\mathrm{MeV})$ & $f_{D}(\mathrm{MeV})$ \\
\hline \hline BLS[6] & $187(10) \pm 37$ & $208(9) \pm 37$ \\
\hline UKQCD[9] & $160_{-6-19}^{+6+53}$ & $185_{-3-7}^{+4+42}$ \\
\hline P-W-C[34 & $204(50)$ & $212(40)$ \\
\hline APE[8] & & $230(30)$ \\
\hline NQRCD[27] & $160(10)$ & \\
\hline HEMCGC[37 & $200(10) \pm 48$ & $250(5) \pm 45$ \\
\hline
\end{tabular}

Table 3

Comparison of other BLS and UKQCD results. Decay constants are in $\mathrm{MeV}$.

\begin{tabular}{|c|c|c|}
\hline & BLS[6] & UKQCD[9] \\
\hline \hline$f_{B_{s}}$ & $207(9) \pm 40$ & $194_{-5-9}^{+6+62}$ \\
\hline$f_{D_{s}}$ & $230(7) \pm 35$ & $212_{-4-7}^{+4+46}$ \\
\hline$f_{B_{s}} / f_{B}$ & $1.11(2) \pm .05$ & $1.22_{-.03}^{+.04}$ \\
\hline$f_{D_{s}} / f_{D}$ & $1.11(2) \pm .05$ & $1.18_{-.02}^{+.02}$ \\
\hline
\end{tabular}

Table 2 shows results from various groups for $f_{B}$ and $f_{D}$, and Table 3 compares results from BLS and UKQCD for other decay constants and ratios. The results are generally in quite good agreement. At the current level of errors, differences in procedure do not show up significantly here. For example, in the case of the BLS vs. P$\mathrm{W}-\mathrm{C}$ results, different normalizations of the propagating quarks $(\sqrt{1-6 \tilde{\kappa}}$ vs. $\sqrt{2 \kappa})$ tend to cancel the effects of different $f_{B}^{\text {stat }}$ (see sec. 2.2), producing quite similar final results for $f_{B}$. The only significant difference between the BLS and UKQCD results occurs in the ratios, especially $f_{B_{s}} / f_{B}$. This difference can be traced mainly to the fact that UKQCD do not include the static values for the ratios in their analysis: both groups get relatively low values $(1.11,1.14)$ in the static limit. Because of the problems with the static signal, it is not clear at this point which procedure is more reliable.

A result not shown in the tables is the HEMCGC value using full $\mathrm{QCD}(\beta=5.3$, Wilson quarks): $f_{D_{s}}=345(5) \pm 48 \mathrm{MeV}$. I do not understand the reason for this large value compared to the quenched results. HEMCGC suggest that it may be due to the larger lattice spacing in these full QCD calculations.

\section{SEMILEPTONIC FORM FACTORS}

Four groups have recently obtained new results for $D \rightarrow K$ and/or $D \rightarrow K^{*} 38$ 41. On the whole, the results are in agreement with previous work 42, 433. Nice plots of the form factors as functions of $q^{2}$ (the square of the 4-momentum transfer) have been obtained. Unfortunately, the statistical errors are not in general appreciably smaller than in the earlier work. This is presumably due to the inherent noisiness associated with lattice propagators of particles with non-zero three-momentum. Just as for static-light mesons [23, 24, 16, 14, the signal-to-noise ratio for mesons with non-zero momentum falls exponentially because the average of the squared propagator has non-zero overlap with a state which has less than twice the meson energy. In this case, the overlap is with a state of two mesons at rest.

One interesting issue concerns the value of the form factor $A_{2}(0)$ (or the ratio $A_{2}(0) / A_{1}(0)$ ). Because of the size of the errors, all the new and old results agree within $2 \sigma$. The new work has, however, has helped to change the qualitative picture. Initially the ELC group 42 found an $A_{2}(0)$ consistent with zero $(0.19 \pm 0.21$ or, later, $0.4 \pm 0.4)$ and with the initial experiment $(0.0 \pm 0.2 \pm 0.1)$ [45]. On the other hand, the BES [43] result was distinctly different from zero $\left(A_{2}(0) / A_{1}(0)=\right.$ $\left.0.70 \pm 0.16_{-0.15}^{+0.20}\right)$. The recent lattice calculations are now giving an $A_{2}(0)$ bounded away from zero: $A_{2}(0) / A_{1}(0)=0.7 \pm 0.4[38] ; A_{2}(0)=0.67 \pm 0.44$ or $0.72 \pm 0.50$ [39] (on two sets of configurations). A non-zero $A_{2}(0)$ is at present also found by experiment: $A_{2}(0) / A_{1}(0)=0.82 \pm 0.23 \pm 0.11$ [46]; $A_{2}(0)=0.44 \pm 0.09$ [47.

Although ref. [38] takes a look at the extrapolation to the $B$ meson, the other lattice studies to date on heavy-light semileptonic form factors have focussed exclusively on $D$ mesons. The issue of normalization of fields and currents when $a m_{Q} \sim 1$ is thus not as crucial here as for $f_{B}$. Still, it is significant numerically.

Consider the normalization of the lattice vector current for large am. For the local current, $V_{\mu}^{\text {local }}(x)=\bar{\psi}(x) \gamma_{\mu} \psi(x)$, the arguments of refs. 32, 15] for normalizing the quark field would 
just give a normalization factor

$\tilde{Z}_{V}^{\text {local }}=1-6 \tilde{\kappa}+\mathcal{O}\left(g^{2}\right)=2 \tilde{\kappa} e^{\tilde{M}_{1} a}+\mathcal{O}\left(g^{2}\right)$,

where the $\mathcal{O}\left(g^{2}\right)$ terms are expected to be small since the tadpoles are summed by using $\tilde{\kappa}$ and $\tilde{M}_{1}$.

The normalization of the conserved current,

$$
\begin{gathered}
V_{\mu}^{\text {cons }}(x)=\frac{1}{2}\left[\bar{\psi}(x)\left(\gamma_{\mu}-1\right) U_{\mu}(x) \psi(x+\hat{\mu})\right. \\
\left.+\bar{\psi}(x+\hat{\mu})\left(\gamma_{\mu}+1\right) U_{\mu}^{\dagger}(x) \psi(x)\right],
\end{gathered}
$$

is more subtle. In the mean field approximation, it is not however hard to see how the current must be normalized in the limit $a m \rightarrow \infty$. Consider the matrix element

$\sum_{\vec{x}}\left\langle 0\left|\psi(\vec{x}, t) V_{\mu}\left(\overrightarrow{0}, t^{\prime}\right) \bar{\psi}(\overrightarrow{0}, 0)\right| 0\right\rangle$,

and compare the case $V_{\mu}=V_{\mu}^{\text {cons }}$ to $V_{\mu}=V_{\mu}^{\text {local }}$. Because the quark just propagates in the time direction for large $m a$, the difference between $V_{0}^{\text {cons }}$ to $V_{0}^{\text {local }}$ will simply be that the former has an extra factor of $u_{0}$ (the mean field value of the explicit link in its definition) but is missing a factor of $e^{-\tilde{M}_{1} a}$ (since the point splitting allows it to skip one hop in the time direction). We thus expect

$\tilde{Z}_{V}^{\text {cons, } \mu=0} \approx \frac{e^{-\tilde{M}_{1} a}}{u_{0}} \tilde{Z}_{V}^{\text {local }} \simeq 2 \kappa$

Eq. (17) is actually exact; the conserved current obeys a Ward identity which can be used to show that $\sum_{\vec{x}} 2 \kappa V_{0}^{\text {cons }}(\vec{x}, t)$ just counts the total charge 48 .

The spatial components $V_{j}^{\text {cons }}$ are however normalized differently. Comparing eq. (16) for conserved and local currents, we see that the former again has an extra factor of $u_{0}$ but is not missing the factor $e^{-\tilde{M}_{1} a}$, since no hops in the time direction are saved. We thus expect

$\tilde{Z}_{V}^{\text {cons, } \mu=j} \approx \frac{1}{u_{0}} \tilde{Z}_{V}^{\text {local }}=2 \kappa e^{\tilde{M}_{1} a}+\mathcal{O}\left(g^{2}\right)$.

Note that although the $\mu=0$ and $\mu=j$ components of $V_{\mu}^{\text {cons }}$ enter the same Ward identity, this does not guarantee that they are normalized
Table 4

Values of " $Z_{V}^{\text {local" }}$ from Table 7 of ref. [43], and the values as calculated perturbatively using the ideas of refs. [31,14]. $J$ is the heavy-light and $V$ is the light-light vector current, respectively.

\begin{tabular}{|c|c|c|}
\hline matrix elem. & ref. [38 & pert.th. \\
\hline$\left\langle V_{1} V_{1}\right\rangle$ & $0.659(3)$ & 0.70 \\
\hline$\left\langle K J_{0} D\right\rangle$ & $0.87(-)$ & 0.83 \\
\hline$\left\langle K J_{1} D\right\rangle$ & $0.75(1)$ & 0.70 \\
\hline$\left\langle K_{3}^{*} J_{1} D\right\rangle$ & $0.66(2)$ & 0.70 \\
\hline
\end{tabular}

the same. The identity involves the divergence of $V_{\mu}^{\text {cons }}$, rather than $V_{\mu}^{\text {cons }}$ itself, and one would need to take into account the difference between $e^{m a}-1$ and $m a$ for the discrete time derivative.

In the literature, the quantity

$\frac{\left\langle\rho\left|V_{j}^{\text {cons }}\right| 0\right\rangle}{\left\langle\rho\left|V_{j}^{\text {local }}\right| 0\right\rangle}=\frac{\tilde{Z}_{V}^{\text {local }}}{\tilde{Z}_{V}^{\text {cons, } \mu=j}}$,

where $\rho$ is a generic vector state, is sometimes used as a "non-perturbative definition" of $Z_{V}^{\text {local }}$. However, the above arguments show that $\tilde{Z}_{V}^{\text {cons, } \mu=j}$ is not fixed by the Ward identity and that the "non-perturbative $Z_{V}^{\text {locall " should in fact }}$ be roughly independent of the quark mass, unlike the correct $Z_{V}^{\text {local }}$ of eq. (15). Indeed, this rough mass independence is found in simulations [37, 38, 41]. The independence is not evidence for a failure of the Kronfeld-Mackenzie ideas, as is suggested in refs. [38,41. On the contrary, it is evidence in favor of ref. 32].

One can go further. Table 7 of ref. 38] gives the

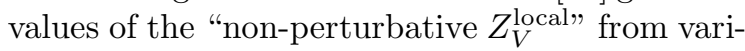
ous comparisons with the conserved current. Using eqs. (17), (18) and a tadpole-improved perturbative calculation of $Z_{V}^{\text {local }}$ with coupling $g_{V}(1 / a)$, I am able to (roughly) reproduce their numbers, as shown in Table 1 . I am neglecting the non-zero 3 -momentum that appears in the last two matrix elements.

Some groups (e.g., refs. 41, 37]) have compared with experiment their results for the vector meson decay constant, $1 / f_{V}$, as a function of $M_{P}^{2} / M_{V}^{2}$ (the square of the pseudoscalar to vector meson mass ratio). Such comparisons can be very misleading since it is known that Wilson quarks get 
much too small a value for the $M_{V}-M_{P}$ splitting for heavy quarks. Thus this is not a good way to test various large- $a m$ normalizations of the vector current.

Finally, the normalization factor $\sqrt{e^{M_{1} a}}$, which is not included in the analysis of ref. [38], is as as large as 1.3 for their heaviest quark masses. Thus the extrapolation of form factors to the $B$ mesons in that paper should be taken as qualitative only.

\section{FORM FACTORS FOR $B \rightarrow K^{*} \gamma$}

The penguin decay $b \rightarrow s \gamma$ [49] provides an excellent test of the Standard Model (SM). It is a short-distance decay at the quark level (the loop with a virtual $t$-quark dominates), so the inclusive rate can be calculated perturbatively. In the $\mathrm{SM}$, the branching ratio $B R(b \rightarrow s \gamma)$ is a slowly varying function of the top quark mass and is essentially independent of the CKM angles in the 3 -generation case. These features make it very sensitive to physics beyond the SM.

The branching ratio for the exclusive decay $B \rightarrow K^{*} \gamma$ has been measured experimentally to be $(4.5 \pm 1.5 \pm 0.9) \times 10^{-5}$ [50]. For theory to make contact with this result, the ratio

$R_{K^{*}} \equiv \frac{\Gamma\left(B \rightarrow K^{*} \gamma\right)}{\Gamma(b \rightarrow s \gamma)}$,

is required. Phenomenological evaluations of $R_{K^{*}}$ vary from $\sim 1 \%$ to $\sim 97 \%$, so the lattice can help out greatly here.

In leading logarithmic order one only one needs to compute the form factors $T_{i}\left(q^{2}\right)$, defined by

$$
\begin{aligned}
&\left\langle K^{*}(k)\left|\bar{s} \sigma^{\mu \nu} q_{\nu} \frac{\left(1+\gamma_{5}\right)}{2} b\right| B(p)\right\rangle= \\
& \sum_{i=1}^{3} c_{i}^{\mu}(p, k) T_{i}\left(q^{2}\right),
\end{aligned}
$$

where the operator on the LHS arises from the short distance expansion of the penguin loop, $q \equiv p-k$ is the photon momentum, and the definitions of the coefficients $c_{i}^{\mu}$ can be found for example in refs. [51,52]. The form factors obey $T_{1}(0)=T_{2}(0)$, and $T_{3}$ does not contribute for a real photon, so $R_{K^{*}}$ is determined by $T_{1}(0)$.

After a first look on the lattice [53], two evaluations of the form factors have been attempted recently 51,522. An extrapolation to the $B$ mass is required as usual. The two groups differ in how they perform the extrapolations. BHS 51 check that pole dominance relates $T_{1}(0)=T_{2}(0)$ to $T_{2}\left(q_{\max }^{2}\right)$, then extrapolate $T_{2}\left(q_{\max }^{2}\right)$ to the $B$ using the relation derived from heavy quark effective theory:

$\sqrt{M_{P}} T_{2}\left(q_{\max }^{2}\right) \sim A+\frac{B}{M_{P}}$,

and finally use pole dominance to get $T_{2}(0)$ from $T_{2}\left(q_{\max }^{2}\right)$ at the $B$. UKQCD [52] calculate $T_{1}(0)$ directly on the lattice (with some mild interpolation) and then extrapolate $T_{1}(0)$ linearly in $1 / M_{P}$ to the $B$.

Each method has some disadvantages: BHS use pole dominance over a wider range $\left(q_{\max }^{2} / M_{B}^{2} \approx\right.$ $0.65)$ than can be checked with the current lattice data $\left(q_{\max }^{2} / M_{P}^{2} \lesssim 0.3\right)$. UKQCD do a "blind" (i.e., without theoretical guidance) extrapolation of $T_{1}(0)$ to the $B$ mass. Obviously I prefer the BHS approach, but both methods are plausible. The difference in the final results gives some measure of the systematics: BHS get $T_{1}(0)=$ $0.10 \pm 0.01 \pm 0.03 ;$ UKQCD get $T_{1}(0)=0.15_{-.04}^{+.05}$ (assuming approximate independence of the spectator quark mass).

Using their result for $T_{1}(0)$, BHS find $R_{K^{*}}=$ $(6.0 \pm 1.2 \pm 3.4) \%$. Combined with the experimental $B R$ for $B \rightarrow K^{*} \gamma$, this produces a $1 \sigma$ bound which is, unfortunately, uninteresting: $m_{t} \gtrsim 100$ $\mathrm{GeV}$. However, because $B R(b \rightarrow s \gamma)$ is a slowly varying function of $m_{t}$, it would not take much improvement in the lattice and/or experimental results to produce a rather stringent bound.

\section{RECENT DEVELOPMENTS ON $B_{K}$}

In the past year, there has been considerable progress in the computation of $B_{K}$, the $K^{0}$ $\bar{K}^{0}$ mixing parameter. First of all, the calculations of the $\mathcal{O}\left(g^{2}\right)$ perturbative corrections to all relevant lattice operators have been completed [54 58]. These include 4-quark operators of the following types: gauge-noninvariant (Landau gauge) "unsmeared" $\left(2^{4}\right)$ [55, 57,58] and "smeared" $\left(4^{4}\right)$ [56,58], and gauge-invariant $\left(2^{4}\right.$ with links) [54,57]. Without the perturbative corrections, results for $B_{K}$ from different operators 
Figure 14. Comparison [60] of $B_{K}$ before (open symbols) and after (solid symbols) renormalization correction. The superscript "inv" denotes gauge-invariant operators; "non-inv," unsmeared Landau-gauge operators.

differ by as much as $\sim 15 \%(\sim 7 \sigma)$ at $\beta=6.0$ [59.60] and as much as $\sim 7 \%(\sim 5 \sigma)$ at $\beta=6.2$ [4]. With the perturbative corrections included, results with different operators agree within errors [59, 60, 何. An example is shown in Fig. 14, taken from ref. 60]. Note that with the bare lattice coupling the disagreement between different operators is reduced but not removed [59]; full agreement occurs when one uses an improved ("boosted") coupling à la Lepage and Mackenzie [15]. (Tadpole improvement is not used here because various operators involve different numbers of links.) The results [61,62, 60] also agree between groups.

The second advance involves the comparison of quenched and full QCD results for $B_{K}$. At the level of the statistics $(\sim 2 \%)$, no difference is found [60,63. The two dynamical quark masses in the full QCD simulations are degenerate and roughly equal to the average quark mass in the kaon $\left(m_{q} \approx m_{s} / 2\right)$. One can easily estimate, using the known chiral logarithms 664,65, how large a difference one would expect in the full theory between the simulated case and the physical situation with $m_{d} \approx m_{u} \approx 0$. The result is a difference of only $3 \%$ to $4 \%$ 国. This of course needs to be checked in simulations. Unfortunately, the quenched theory cannot be used to estimate the difference between a $B_{K}$ with degenerate quarks and one with a light $m_{d}$. Quenched chiral perturbation theory shows [65] that the chiral logs for a $B_{K}$ with non-degenerate quarks (in contrast to the degenerate case) are different in the quenched and full theories.

The third advance is the understanding of the lattice spacing errors. At Lattice '91, the $B_{K}$ data 62] showed a strong dependence on $a$, and it was unclear how to extrapolate to the continuum: A linear extrapolation in $a$ gave $B_{K} \alpha_{s}^{-2 / 9} \equiv \hat{B}_{K}=$ $0.66(6)$; a quadratic, $\hat{B}_{K}=0.78(3)$. This difference dominated the systematic errors. Sharpe [4] has now shown that the lattice spacing errors are $\mathcal{O}\left(a^{2}\right)$. The basic idea is simple: the staggered fermion action has corrections only at $\mathcal{O}\left(a^{2}\right)$. So corrections of $\mathcal{O}(a)$ to $B_{K}$ could come only from the dimension- 6 weak operators themselves, through mixing with dimension-7 operators. However, a detailed enumeration shows that no such operators exist with all the right symmetries: hypercubic group, flavor symmetry, and individual axial rotations of each flavor.

Once one knows the lattice spacing errors are $\mathcal{O}\left(a^{2}\right)$, it is straightforward to include the perturbative corrections and extrapolate existing data of Gupta, Kilcup and Sharpe 62] to the contin-

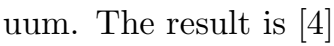

$$
\begin{gathered}
B_{K}(\mathrm{NDR}, 2 \mathrm{GeV})=0.616 \pm 0.020 \pm 0.017 \\
\hat{B}_{K}=0.825 \pm 0.027 \pm 0.023
\end{gathered}
$$

where the continuum $\alpha_{s}$ with $\Lambda \frac{(4)}{\mathrm{MS}}=300 \mathrm{MeV}$ is used to obtain the second equation. The only errors not included above are those due to quenching and to the use of degenerate quarks. As discussed above, the quenched and full theories agree for degenerate quarks at a scale roughly that of the quenched $\beta=6.0\left(a^{-1} \sim 2 \mathrm{GeV}\right)$. One needs however to check that the theories continue to agree at smaller lattice spacings. In addition, the effects of non-degenerate quarks in the full theory need to be studied. However, given the size of the chiral logs, I would be surprised if this amounted to more than a $5 \%$ or $6 \%$ error. Thus if one at most doubles the systematic errors in eqs. (22), (23), one has a result which is likely to 
be very reliable. This is a major accomplishment of lattice QCD.

I'd like to briefly mention one other result which has implications for light-light weak matrix elements. Kuramashi and collaborators [66] have obtained results for $\pi-\pi$ scattering in QCD. Their method makes possible the first calculation of the $I=0$ scattering length. The same method may ultimately allow the computation of the $\Delta I=1 / 2$ $K \rightarrow \pi \pi$ weak amplitude, a computation which has bedeviled some of us in lattice gauge theory over the past ten years.

\section{CONCLUSION}

The basic results have already been summarized in the introduction. I would just like to add the observation that we owe a great debt to Lepage and Mackenzie for showing how to understand and control lattice perturbation theory 15 . This has made possible many of the advances discussed above.

\section{ACKNOWLEDGEMENTS}

I thank C. Allton, S. Collins, C. Davies, T. DeGrand, T. Draper, R. Gupta, S. Güsken, E. Eichten, B. Hill, A. Kronfeld, J. Labrenz, D. Leinweber, P. Mackenzie, G. Martinelli, M. Okawa, A. Pich, D. Richards, C. Sachrajda, H. Shanahan, S. Sharpe, A. Soni, R. Sommer, N. Stella, and A. Ukawa for useful discussions. This work was supported in part by the US Department of Energy under grants DE2FG02-91ER40628 and DEFG0393-ER25186.

\section{REFERENCES}

1. See, for example, A. Buras, MPI-Ph/93-52 (7/93); M. Lusignoli, L. Maiani, G. Martinelli and L. Reina, Nucl. Phys. B369 (1992) 139; A. Pich, in proceedings of the 4th Conference on the Intersections Between Particle and Nuclear Physics, Tucson, AZ (5/91), p. 634.

2. E. Eichten, Nucl. Phys. B (Proc. Suppl.) 4 (1988) 170.

3. I do not discuss the Isgur-Wise function; see instead R. Kenway, these proceedings.

4. S. Sharpe, these proceedings.
5. A. Duncan, E. Eichten, J. Flynn, B. Hill, and $\mathrm{H}$. Thacker, these proceedings.

6. C. Bernard, J. Labrenz and A. Soni, UW/PT93-06 (7/93), to be published in Phys. Rev. D.

7. C.R. Allton, et al., Rome prep. 93/928 (revised 8/93).

8. C. Allton for APE, these proceedings.

9. R.M. Baxter et al., (UKQCD collab.), Edinburgh Preprint: 93/526; D.S. Henty for UKQCD, these proceedings.

10. C. Alexandrou et al., CERN-TH 6692/92.

11. S. Hashimoto, these proceedings.

12. G.S. Bali and K. Schilling, Phys. Rev. D47 (1993) 661. For values of $\beta$ not included in the above, I have interpolated the string tension using the two-loop asymptotic scaling formula with an improved coupling.

13. E. Eichten and B. Hill, Phys. Lett. 234B (1990) 511 and Phys. Lett. 240B 193 (1990); Ph. Boucaud, C.L. Lin and O. Pène, Phys. Rev. D40 (1989) 1529 and Phys. Rev. D41 (1990) 3541(E).

14. O.F. Hernandez and B.R. Hill, Phys. Lett. 289B 417 (1992); A. Borrelli and C. Pittori, Nucl. Phys. B385 (1992) 502.

15. G.P. Lepage and P.B. Mackenzie, Phys. Rev. D48 (1993) 2250.

16. A. Duncan, E. Eichten, G. Hockney, and H. Thacker, Nucl. Phys. B (Proc. Suppl.) 26 (1992) 391.

17. A. Duncan, E. Eichten, and H. Thacker, Nucl. Phys. B (Proc. Suppl.) 26 (1992) 394; Nucl. Phys. B (Proc. Suppl.) 30 (1993) 441.

18. G. Martinelli and Zhang Yi-Cheng, Phys. Lett. 123B, 433 (1983).

19. Ref. 15] gives many other success stories for tadpole improvement. See D. Leinweber, these proceedings, for an additional nice example.

20. C. Morningstar, these proceedings.

21. B. Hill, these proceedings.

22. B. Hill and O. Hernandez, in preparation.

23. P. Lepage, Nucl. Phys. B (Proc. Suppl.) 26 (1992) 45.

24. C. Bernard, C.M. Heard, J. Labrenz, and A. Soni, Nucl. Phys. B (Proc. Suppl.) 26 (1992) 384.

25. E. Eichten, G. Hockney, and H. Thacker, 
Nucl. Phys. B (Proc. Suppl.) 17 (1990) 529.

26. T. Draper and C. McNeile, these proceedings.

27. C.T.H. Davies for the UKQCD collaboration, poster session, these proceedings.

28. G.P. Lepage and J. Sloan for the NRQCD collaboration, these proceedings; C.T.H. Davies, review talk on NRQCD, these proceedings.

29. L. Maiani, G. Martinelli, and C. Sachrajda, Nucl. Phys. B368 (1992) 281.

30. A. Duncan, et al., Nucl. Phys. B (Proc. Suppl.) 30 (1993) 433.

31. C. Bernard, J. Labrenz, and A. Soni, Nucl. Phys. B (Proc. Suppl.) 30 (1993) 465.

32. P. Mackenzie, Nucl. Phys. B (Proc. Suppl.) 30 (1993) 35; A. Kronfeld, Nucl. Phys. B (Proc. Suppl.) 30 (1993) 445; see also ref.23.

33. A. Kronfeld, these proceedings.

34. S. Güsken for the PSI-Wuppertal-CERN group, these proceedings.

35. G. Martinelli et al., Phys. Lett. 311B (1993) 241.

36. A. Abada et al., Nucl. Phys. B376 (1992) 172 .

37. K. Bitar et al. (HEMCGC collaboration) Phys. Rev. D48 (1993) 370 and FSU-SCRI93-110 (9/93); see also T. DeGrand, 1993 SLAC Summer Institute lectures (COLOHEP 322) and these proceedings.

38. A. Abada et al., LPTENS 93/14, ROME prep. 93/946 (7/93).

39. N. Stella for APE, these proceedings.

40. D. Richards for UKQCD, these proceedings.

41. R. Gupta and T. Bhattacharya, these proceedings; R. Gupta, T. Bhattacharya, and D. Daniel, LAUR-93-3580 (10/93).

42. M. Crisafulli et al., Phys. Lett. 223B (1989) 90; V. Lubicz et al., Nucl. Phys. B356 (1991) 310 and Phys. Lett. 274B (1992) 415.

43. C. Bernard, A. El-Khadra, and A. Soni, Nucl. Phys. B (Proc. Suppl.) 9 (1989) 189; Phys. Rev. D43 (1991) 2140; and Phys. Rev. D45 (1992) 869.

44. for an introduction to such signal-to-noise arguments, see P. Lepage, in From Actions to Answers, T. DeGrand and D. Toussaint (eds.), (World Scientific, 1990) p. 97.

45. J. Anjos et al. (E691 collab.), Phys. Rev. Lett. 65 (1990) 2630.
46. K. Kodama et al. (E653 collab.), Phys. Lett. 274B (1992) 246.

47. M. Witherell, talk at the Lepton-Photon Conference, Cornell, 1993.

48. see, for instance, J. Labrenz, UCLA Ph.D. thesis $(9 / 92)$.

49. see for instance, M. Misiak, Nucl. Phys. B393 (1993) 23 and references therein.

50. D. Ammar et al. (CLEO collab.), Phys. Rev. Lett. 72 (1993) 674.

51. C. Bernard, P. Hsieh, and A. Soni, Wash. U. HEP/93-35 (11/93); A. Soni, talk at this conference.

52. K.C. Bowler et al. (UKQCD), Edinb. prep. (11/93); H.P. Shanahan, these proceedings.

53. C. Bernard, P. Hsieh, and A. Soni, Nucl. Phys. B (Proc. Suppl.) 26 (1992) 347.

54. S.N. Sheard, Nucl. Phys. B314 (1989) 238.

55. S.R. Sharpe, Nucl. Phys. B (Proc. Suppl.) 7A (1989) 255.

56. A. Patel and S. Sharpe, Nucl. Phys. B395(1993) 701.

57. N. Ishizuka and Y. Shizawa, Tsukuba prep. UTHEP-261 (8/93).

58. S. Sharpe and A. Patel, UW/PT-93-1 (10/93).

59. N. Ishizuka et al., Nucl. Phys. B (Proc. Suppl.) 30 (1993) 415.

60. N. Ishizuka et al., Phys. Rev. Lett. 71 (1993) 24.

61. G.W. Kilcup et al., Phys. Rev. Lett. 64 (1990) 25.

62. S.R. Sharpe et al., Nucl. Phys. B (Proc. Suppl.) 26 (1992) 197.

63. G.W. Kilcup, Phys. Rev. Lett. 71 (1993) 1677.

64. J. Bijnens, H. Sonada, and M. Wise, Phys. Rev. Lett. 53 (1985) 2367.

65. S.R. Sharpe, Phys. Rev. D46 (1992) 3146.

66. Y. Kuramashi, M. Fukugita, H. Mino, M. Okawa, and A. Ukawa, these proceedings. 
This figure "fig1-1.png" is available in "png" format from: http://arxiv.org/ps/hep-lat/9312086v1 
This figure "fig2-1.png" is available in "png" format from: http://arxiv.org/ps/hep-lat/9312086v1 
This figure "fig1-2.png" is available in "png" format from: http://arxiv.org/ps/hep-lat/9312086v1 
This figure "fig2-2.png" is available in "png" format from: http://arxiv.org/ps/hep-lat/9312086v1 
This figure "fig1-3.png" is available in "png" format from: http://arxiv.org/ps/hep-lat/9312086v1 
This figure "fig2-3.png" is available in "png" format from: http://arxiv.org/ps/hep-lat/9312086v1 